\title{
Chromosomal characteristics and evolutionary relationships of the Palearctic black fly Simulium carthusiense (Diptera: Simuliidae)
}

\author{
Peter H. ADLER ${ }^{1}$ and GunTHER SEITZ ${ }^{2}$ \\ ${ }^{1}$ Entomology Program, Clemson University, Clemson, SC 29634-0310 USA; e-mail: padler@clemson.edu \\ ${ }^{2}$ District Government of Lower Bavaria, Regierungsplatz 540, 84028 Landshut, Germany; e-mail: gunther.seitz@reg-nb.bayern.de
}

Key words. Diptera, Simuliidae, Simulium carthusiense, Simulium urbanum, aquatic insects, black flies, polytene chromosomes, Spain

\begin{abstract}
The giant, polytene chromosomes of Simulium carthusiense Grenier \& Dorier, 1959 were mapped, and all rearrangements were resolved relative to the standard banding sequence for the $S$. vernum group. The species is chromosomally cohesive from Austria to Spain, and is characterized by a chromocenter, two unique fixed inversions, 10 unique autosomal polymorphisms, and undifferentiated sex chromosomes. Rare individuals (3\%) have two types of supernumerary chromosomes, representing the third example of a simuliid species that carries two different supernumeraries in the same individuals. Band-sequence comparisons with chromosomal outgroups indicate that $S$. carthusiense is the sister species of a clade that includes $S$. beltukovae (Rubtsov, 1956), the $S$. cryophilum complex, and S. urbanum Davies, 1966.
\end{abstract}

\section{INTRODUCTION}

The Simulium vernum group provides numerous examples of the taxonomic difficulties in the family Simuliidae (Ilmonen et al., 2009). It is the second largest species group of black flies in the world, consisting of about 130 nominal species, $80 \%$ of which are represented in the Palearctic Region (Adler \& Crosskey, 2014). Members of the S. vernum group are structurally similar and often can be identified in only one or two life stages. The taxonomy and systematics of the group, however, have been aided through studies of the banding patterns in the giant chromosomes of the larval silk glands. These giant, polytene chromosomes have revealed cryptic species, defined species limits, and resolved evolutionary relationships in the group (Brockhouse, 1985; Hunter, 1987). About 26 Palearctic species in the $S$. vernum group have been examined chromosomally (e.g., Chubareva \& Petrova, 2008), but only about half of these have been resolved relative to an established reference map (Brockhouse, 1985; Hunter, 1987; Bass \& Brockhouse, 1990; Adler et al., 2004; Ilmonen et al., 2009).

The polytenes of the Simuliidae are expressed as 3, rarely 2, haploid submetacentric chromosomes (I, II, and III) each with a long (L) and short (S) arm on either side of the centromere (Rothfels, 1979). Chromosomal landmarks are consistently found within an arm and, therefore, aid arm recognition (Rothfels et al., 1978). A primary nucleolar organizer can appear anywhere in the complement, though its location is consistent at some taxonomic level (Adler et al., 2004). Chromosomal variability within and among species can be assessed by evaluating structural rearrangements, primarily inversions and bands of differential thickness (heterobands). These rearrangements can be fixed, autosomally polymorphic, or linked to the X or Y chromosome (Bedo, 1977; Adler et al., 2010). A standard chromosomal map for a taxon (e.g., species group) typically represents the most central banding sequence in the group (e.g., Brockhouse, 1985), allowing the banding patterns of all members of the taxon to be compared. Rearrangements, relative to the standard map, are considered to represent unique events (Rothfels, 1979), and can provide phylogenetic information when they are shown, by outgroup comparison, to be uniquely shared and derived from an ancestral condition (Adler \& Huang, 2011).

Simulium carthusiense Grenier \& Dorier, 1959, a typical member of the $S$. vernum group, was described from females, males, pupae, and larvae collected in the French Alps, and was considered conspecific with material from the Pyrenees of France and from Andalusia, Spain, collected at elevations of 300-2500 m (Grenier \& Dorier, 1959). It subsequently has been found in Andorra, Austria, Czech Republic, Germany, Italy, Poland, Slovakia, Switzerland, and Ukraine (Adler \& Crosskey, 2014). The preimaginal stages develop in swift mountain streams, and pupation occurs in June, producing one generation per year (Knoz, 1965).

We evaluate the polytene chromosomes of Simulium carthusiense against the standard map of Brockhouse (1985) for the $S$. vernum group, mapping all structural rearrangements, identifying unique features, and establishing evolutionary relationships with closely related species. The resulting cytophylogeny is the first, based on a cladistic approach, for any species in the $S$. vernum group.

\section{MATERIAL AND METHODS}

Larvae of $S$. carthusiense were hand collected from 4 streams in Austria and Spain (Table 1) and were fixed in 3 changes of $1: 3$ acetic ethanol. In Austria, larvae and pupae were collected with those of Prosimulium rufipes (Meigen, 1830) at Site 1 and with $P$. rufipes and S. bertrandi Grenier \& Dorier, 1959 at Sites 2 and 3. These sites were in epirhithral, oligosaprobic, alpine, karstic streams with boulders. In Spain (Site 4), larvae and pupae were collected with those of $S$. cryophilum cytoform "A" (IIIL-19= rr sequence of Hunter, 1987) and larvae of S. urbanum (chromo- 


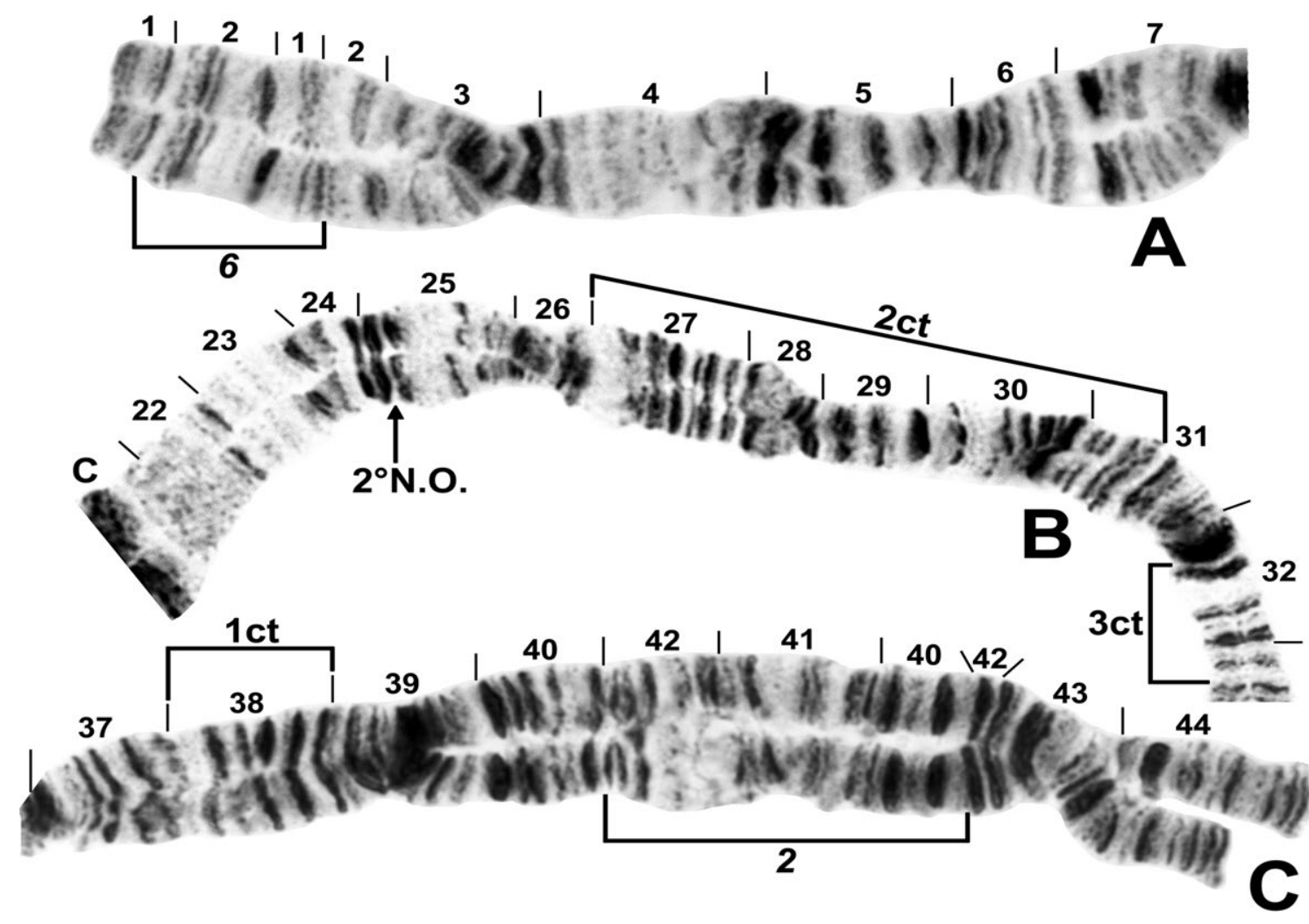

Fig. 1. Chromosome I of Simulium species. A - IS end of S. carthusiense, showing IS-6 sequence. B - IL base of S. cryophilum cytoform "A" (female larva from Vindrarp, Sweden), representing the standard sequence with the banding pattern identical to that of $S$. carthusiense; breakpoints of IL-2ct and IL-3ct are indicated by brackets, and the location of a secondary nucleolar organizer ( $\left.2^{\circ} \mathrm{N} . \mathrm{O} . \mathrm{ct}\right)$ by an arrow; $\mathrm{C}=$ centromere. $\mathrm{C}-\mathrm{IL}$ end of $S$. carthusiense, showing $I L-2$ sequence; breakpoints of IL-1ct are indicated by brackets.

somally typical, Adler et al., 1999), the latter species representing the first record for Spain and a southward range extension of about $1500 \mathrm{~km}$. Site 4 was an aggregate of hypocrenal spring brooks in the Sierra Nevada in the vicinity of the Andalusian site where Grenier \& Dorier (1959) found S. carthusiense.

The polytene chromosomes and gonads of larvae were prepared using the Feulgen procedure (Rothfels \& Dunbar, 1953; Adler et al., 2004). Representative chromosomes from female larvae collected at Site 2 (Figs 1, 2A, 3) and Site 4 (Fig. 4) and male larvae collected at Site 1 (Fig. 2B) were photographed on an Olympus BX40 compound microscope and assembled into maps, using Adobe ${ }^{\circledR}$ PhotoShop ${ }^{\circledR}$ Elements 8 . Larval carcasses from which chromosomes were prepared, plus photographs of selected chromosome preparations, were deposited in the Clemson University Arthropod Collection.

Section numbering on our maps follows that of Brockhouse (1985). Chromosomal sequences of all 33 larvae were compared

TABLE 1. Collection sites for chromosomally analyzed larvae of Simulium carthusiense.

\begin{tabular}{ccccc}
\hline $\begin{array}{l}\text { Site } \\
\text { No. }\end{array}$ & Location & $\begin{array}{c}\text { Latitude, } \\
\text { longitude }\end{array}$ & $\begin{array}{c}\text { Elevation } \\
\text { (m a.s.1.) }\end{array}$ & Date \\
\hline \multirow{2}{*}{1} & AUSTRIA, & $47^{\circ} 34^{\prime} 31^{\prime \prime} \mathrm{N}$ & 785 & 28 June 2013 \\
& Haindlkarbach & $14^{\circ} 36^{\prime} 51^{\prime \prime} \mathrm{E}$ & & \\
2 & AUSTRIA, & $47^{\circ} 46^{\prime} 31^{\prime \prime} \mathrm{N}$ & 985 & 27 June 2013 \\
& Moderbach & $15^{\circ} 06^{\prime} 13^{\prime \prime} \mathrm{E}$ & & \\
\multirow{2}{*}{3} & AUSTRIA, & $47^{\circ} 46^{\prime} 36^{\prime \prime} \mathrm{N}$ & 980 & 27 June 2013 \\
& Rothausbach & $15^{\circ} 05^{\prime} 41^{\prime \prime} \mathrm{E}$ & & \\
4 & SPAIN, Fuente Alta, & $37^{\circ} 06^{\prime} 20^{\prime \prime} \mathrm{N}$ & 2200 & 15 May 2010 \\
& Sierra Nevada & $03^{\circ} 24^{\prime} 27^{\prime \prime} \mathrm{W}$ & & \\
\hline
\end{tabular}

band-for-band with the standard map established by Brockhouse (1985) for the $S$. vernum group. All fixed and common rearrangements and supernumerary (B) chromosomes were photographed. Infrequent rearrangements - those in $3 \%$ or less of all homologues (Table 2) - were mapped, with their precise breakpoints and locations indicated by brackets and arrows (Figs 1-3). Numbering of novel, fixed inversions follows the sequence of Hunter (1987) and Seitz \& Adler (2009); unique polymorphisms are identified with a

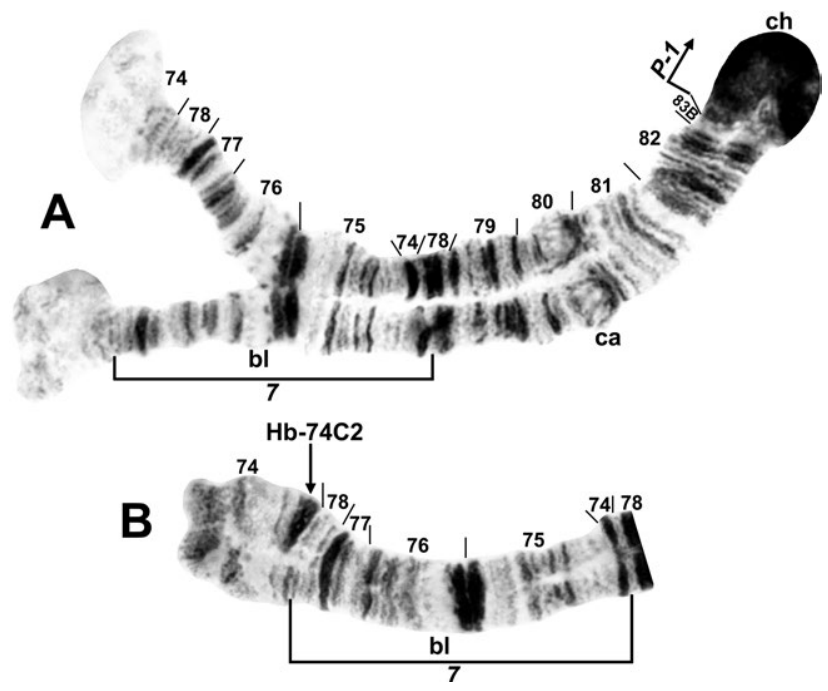

Fig. 2. IIIS of Simulium carthusiense. A - IIIS-7 and IIIP-1 sequences; $\mathrm{bl}=$ blister, $\mathrm{ca}=$ capsule, $\mathrm{ch}=$ chromocenter. $\mathrm{B}-$ IIIS end showing IIIS-7 sequence and heterozygous expression of $\mathrm{Hb}-$ $74 \mathrm{C} 2$ (arrow). 


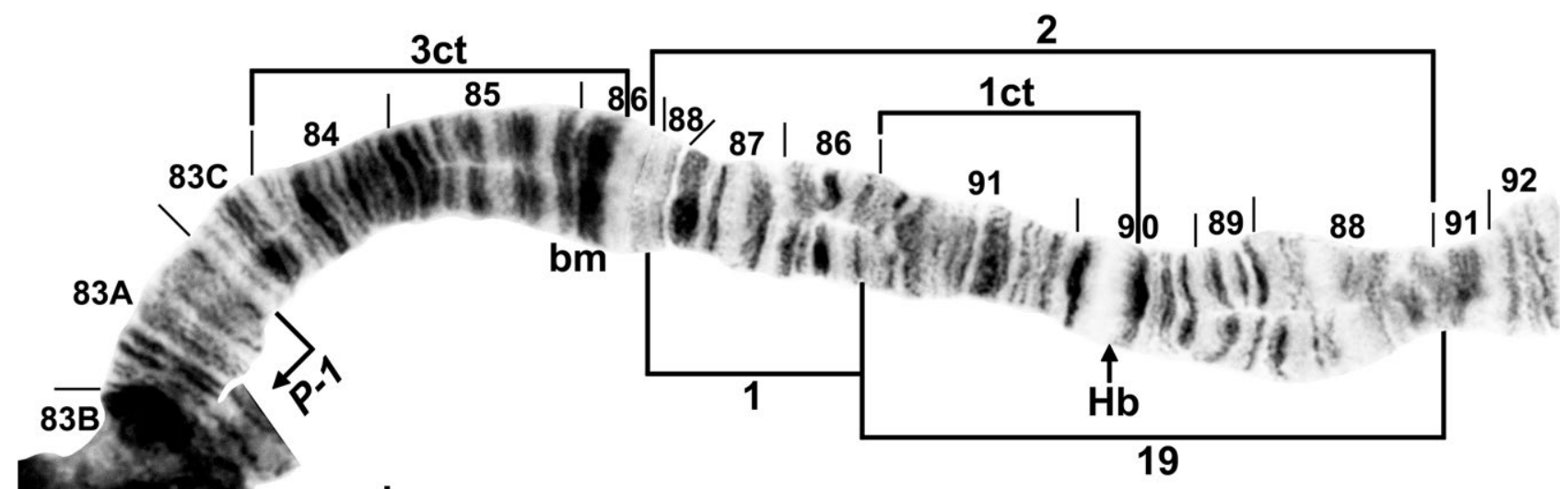

Fig. 3. IIIL of Simulium carthusiense, showing IIIL-19.1 (qq) and IIIP-1 sequences. Breakpoints of IIIL-2 (t sequence), IIIL-1ct, IIIL-2ct, and IIIL-3ct are indicated by brackets, and locations of secondary nucleolar organizer ( $2^{\circ}$ N.O.) and Hb-90C2ct (Hb) by arrows; $\mathrm{bm}=$ basal marker, $\mathrm{ch}=$ chromocenter.

TABLE 2. Frequency of all chromosomal rearrangements for Simulium carthusiense relative to the standard sequence for the S. vernum group.

\begin{tabular}{lcccc}
\hline & Site 1 & Site 2 & Site 3 & Site 4 \\
\hline Female : Male & $1: 2$ & $21: 1$ & $1: 4$ & $2^{1}: 1$ \\
IS-6 & 1.00 & 1.00 & 1.00 & 1.00 \\
IS 2 ${ }^{\circ}$ N.O.ct ${ }^{2}$ & & 0.02 & & \\
IL-2 & 1.00 & 1.00 & 1.00 & 1.00 \\
IL-1ct & & & 0.10 & \\
IL-2ct & & 0.02 & 0.20 & \\
IL-3ct & & 0.02 & & \\
IL 2 ${ }^{\circ}$ N.O.ct & & 0.02 & & \\
IIIS-7 & 1.00 & 1.00 & 1.00 & 1.00 \\
IIIS Hb-74C2 & 0.33 & 0.02 & 0.20 & \\
III-P1 & 1.00 & 1.00 & 1.00 & 1.00 \\
IIIL-19.1 (= q) & 1.00 & 0.98 & 1.00 & 1.00 \\
IIIL-2 (= t) & & 0.02 & & \\
IIIL-1ct & & 0.02 & & \\
IIIL-2ct & & 0.02 & & \\
IIIL-3ct & & 0.02 & & \\
IIIL 2 ${ }^{\circ}$ N.O.ct & & 0.02 & & \\
IIIL Hb-90C2ct & & 0.02 & & \\
B chromosomes & & & & \\
Chromocenter & 1.00 & 1.00 & 1.00 & 1.00 \\
\hline I female & & &
\end{tabular}

${ }^{1} 1$ female larva infected with the microsporidium Amblyospora possibly bracteata.

${ }^{2}$ Secondary nucleolar organizer ( $2^{\circ}$ N.O. $)$ in section 17 at the A/B junction of Brockhouse (1985). species modifier (ct); thus, ct $=$ S. carthusiense (e.g., IIIL-1ct). Italicized rearrangements were fixed; nonitalicized rearrangements were polymorphic. Terminology for B chromosomes follows that of Brockhouse et al. (1989).

To examine the evolutionary relationships of $S$. carthusiense, we compared its banding patterns with the sequences of related

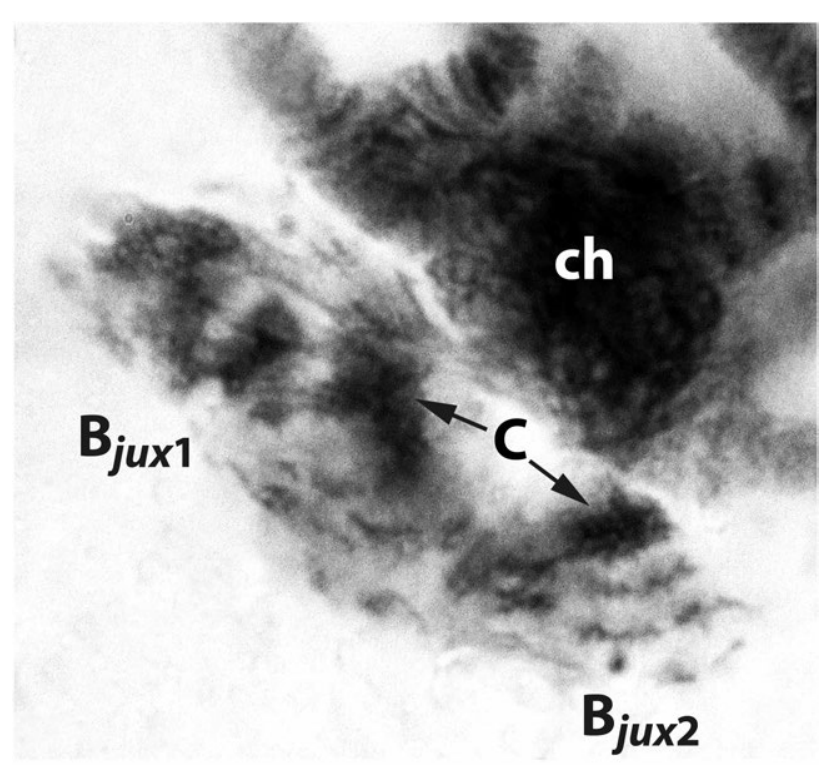

Fig. 4. Two types of supernumerary chromosomes $\left(\mathrm{B}_{j u x \mathrm{x}}\right.$ and $\mathrm{B}_{j u x 2}$ ) of Simulium carthusiense, attached by their centromeres (C) to the chromocenter (ch) of the 3 typical chromosomes. 
species in the $S$. vernum group, specifically those species that share the pericentric IIIP-1 fixed inversion first recognized by Hunter (1987). Where possible, we compared band sequences of these species with the relevant sequences in two outgroups, the S. canonicolum group (Golini \& Rothfels, 1984) and S. ruficorne Macquart, 1838 (Bedo, 1989). Our cytophylogeny, therefore, is based on unique, shared chromosomal rearrangements derived from a common ancestor.

\section{RESULTS AND DISCUSSION}

\section{Chromosomal characteristics}

All larvae (25 females, 8 males) had the typical haploid complement of $n=3$ chromosomes found in about $96 \%$ of the more than 500 cytologically examined species of simuliids (Adler et al., 2010). The chromosomal homologues were paired along $76-85 \%$ of their lengths, comparable to the degree of pairing seen in other temperate species of black flies in May and June (Rothfels \& Featherston, 1981), and about three times the extent of pairing in $S$. vernum Macquart, 1826 (Adler et al., 1999). The three chromosomes were consistently united at their centromeres in a large, darkly staining chromocenter. More than $12 \%$ of all studied simuliids have a chromocenter (Adler et al., 2010), which aids rapid species diagnosis. The primary nucleolar organizer was located in the base of IS, the typical location for the S. vernum group (Brockhouse, 1985; Hunter \& Connolly, 1986; Hunter, 1987). In addition to the homozygous primary nucleolus in all larvae, three secondary nucleolar organizers microscopically identical to the primary nucleolus occurred as rare heterozygotes - each in $1.5 \%$ of all homologues (Table 2). Secondary nucleolar organizers in the Simuliidae are frequent across species, but infrequent within species. Multiple nucleolar sites are postulated to occur in the genome, with one dominant to the exclusion of others in a species; incomplete suppression, however, might result in nucleolar expression at another site (Bedo, 1978).

All larvae were fixed for $I S-6, I L-2, I I I S-7$, and IIIP-1 inversions (Figs 1-3). Thirteen additional rearrangements, all autosomal, were found among the 33 larvae (Table 2). IIIL-19.1 (q sequence of Hunter, 1987) was nearly fixed; one Moderbach female larva lacked IIIL-19.1 in one homologue, instead carrying IIIL-2 (t sequence of Hunter, 1987); thus, the larva was a IIIL-qt heterozygote. All but one of the 13 inversions were paracentric. Only IIIP-1 was pericentric, resulting in shortening of the IIIS arm by about $6 \%$ and lengthening of the IIIL arm by about $2 \%$. Three secondary nucleolar organizers, one heteroband ( $\mathrm{Hb}-90 \mathrm{C} 2 \mathrm{ct})$, and six autosomal inversions were unique to $S$. carthusiense. Inversion heterozygosity per larva was low -0.3 across all sites. Only three polymorphisms were found at more than one collection site: the nearly fixed IIIL-19.1 (Fig. 3); IIIS Hb-74C2 (Fig. 2B), which occurred heterozygously in two female and three male larvae; and IL-2ct (Fig. 1B), which was found at two sites less than $1 \mathrm{~km}$ apart. Of the two heterobands, only IIIS Hb-74C2 occurred in more than one larva. Heterobands, reflecting enhanced DNA content, are found in many species of the Simuliidae (Bedo, 1978), including other members of the $S$. vernum group (Hunter,

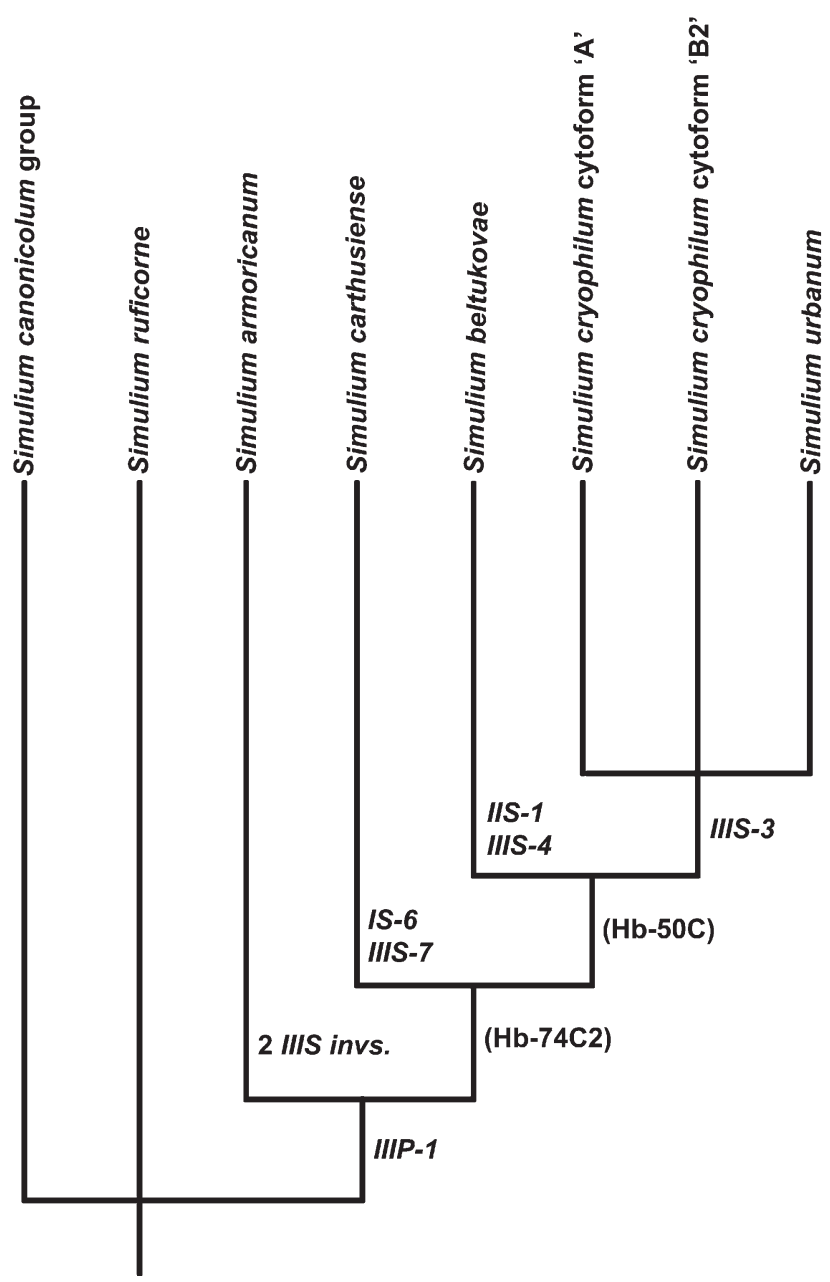

Fig. 5. Cytophylogeny of chromosomally studied members of the $S$. vernum group, which share the pericentric IIIP-1 inversion of Hunter (1987), based only on uniquely shared rearrangements; parentheses indicate that the rearrangement is polymorphic; italics indicate that the inversion is fixed.

1987). None of the rearrangements in S. carthusiense were linked to the $\mathrm{X}$ or Y chromosomes; the sex chromosomes, therefore, were microscopically undifferentiated $\left(\mathrm{X}_{0} \mathrm{Y}_{0}\right)$. About three-quarters of the 26 studied Palearctic species in the $S$. vernum group have undifferentiated sex chromosomes (Bass \& Brockhouse, 1990; Ilmonen et al., 2009; Seitz \& Adler, 2009).

One female larva (Site 4) had polytenized supernumerary (B) chromosomes expressed as a large, flocculent mass. Two heavy centromere bands, each representing a distinct telocentric B chromosome, were attached to the chromocenter of the three chromosomes of the A complement (Fig. 4); the B chromosomes were detached from the chromocenter in about $10 \%$ of the nuclei. Thus, $S$. carthusiense, like $S$. juxtacrenobium Bass \& Brockhouse, 1990 and S. costatum Friederichs, 1920, has two types of $\mathrm{B}$ chromosomes, one of which is equivalent to $\mathrm{B}_{j u x 2}$ and the other is either equivalent to $\mathrm{B}_{j u x 1}$ or unique (Brockhouse et al., 1989). The discovery of B chromosomes in S. carthusiense, which are shared with several other members of the S. vernum group (Brockhouse, 1985; Brockhouse et al., 1989), indicates a deeper common ancestry for the 
Bs. Rothfels (in Brockhouse et al., 1989) suggested that all known B chromosomes in the family Simuliidae share a common origin.

We conclude that our samples of $S$. carthusiense represent a single species chromosomally distinct from all other cytologically analyzed species of the $S$. vernum group.

\section{Phylogenetic relationships}

IS-6 and IIIS-7 are unique to S. carthusiense, and both sequences are derived, relative to the ancestral condition in our outgroups. $I L-2$ and IIIL-19 are shared with many members of the $S$. vernum group in the Holarctic Region, whereas IIIP-1, IIIL-1, and IIIL-2 (Fig. 3) are shared with a restricted group of European species, including $S$. armoricanum Doby \& David, 1961, S. bavaricum Seitz \& Adler, 2009, S. beltukovae (Rubtsov, 1956) - chromosomally studied as S. carpathicum (Knoz, 1961) - the S. cryophilum complex, and S. urbanum Davies, 1966 - chromosomally studied as "B1" - (Hunter, 1987; Seitz \& Adler, 2009). Of these five inversions, only IIIP-1 could be determined by outgroup comparison as derived from the ancestral sequence; the remaining four inversions were in areas of the complement too scrambled for reliable comparison with outgroup sequences. We determined that all previously published rearrangements in IIS and IIIS (Hunter, 1987), which are used in our cytophylogeny, are derived from the ancestral condition (Fig. 5). IIIS Hb-74C2 of S. carthusiense is shared with $S$. beltukovae, $S$. cryophilum cytoforms "A" and "B2", and S. urbanum (Hunter, 1987). Simulium carthusiense is the sister species of a clade united by IIS Hb-50C of Hunter (1987), which includes S. beltukovae, the $S$. cryophilum complex, and S. urbanum.

Although the entire $S$. vernum group represents a welldefined clade based on structural characters, particularly the unique shape of the gonostylus, structural characters for inferring relationships within the group are limited (Adler et al., 2004). Our phylogeny reveals a clade of closely related species within the group, defined by the unique pericentric IIIP-1 inversion, demonstrating that chromosomal characters can provide species-level resolution of relationships. Unlike a majority of closely related black flies (Rothfels, 1989), however, differentiation of sex chromosomes has not played a role in the evolution of the IIIP-1 clade; only $S$. armoricanum has differentiated sex chromosomes (Bass $\&$ Brockhouse, 1990). The IIIP-1 clade is biogeographically cohesive; all species are found in Western Europe, and are concentrated in the area from Spain to Germany.

ACKNOWLEDGEMENTS. We thank the Gesäuse National Park Administration, Austria, for organization and support during the International Fountain and Creek Week. We are grateful to C.E. Beard for technical assistance. This work was funded, in part, by National Science Foundation award DEB-0933218. This is Technical Contribution No. 6246 of the Clemson University Experiment Station, and is based on work supported in part by NIFA/ USDA under project number SC-1700433.

\section{REFERENCES}

Adler P.H. \& Crosskey R.W. 2014: World Blackflies (Diptera: Simuliidae): A Comprehensive Revision of the Taxonomic and
Geographical Inventory [2014]. 122 pp. http://www.clemson. edu/cafls/biomia/pdfs/blackflyinventory.pdf

AdLeR P.H. \& HuANG Y.T. 2011: Integrated systematics of the Simuliidae (Diptera): evolutionary relationships of the littleknown Palearctic black fly Simulium acrotrichum. - Can. Entomol. 143: 612-628.

Adler P.H., Malmqvist B. \& Zhang Y. 1999: Black flies (Diptera: Simuliidae) of northern Sweden: taxonomy, chromosomes, and bionomics. - Entomol. Scand. 29: 361-382.

Adler P.H., Currie D.C. \& Wood D.M. 2004: The Black Flies (Simuliidae) of North America. Cornell University Press, Ithaca, NY, xv +941 pp. +24 color plates.

Adler P.H., Cheke R.A. \& Post R.J. 2010: Evolution, epidemiology, and population genetics of black flies (Diptera: Simuliidae). - Infection, Genetics Evol. 10: 846-865.

Bass J.A.B. \& Brockhouse C. 1990: A new British species of the Simulium vernum group, with comments on its ecology and life history (Diptera: Simuliidae). — Aquat. Insects 12: 65-84.

Bedo D.G. 1977: Cytogenetics and evolution of Simulium ornatipes Skuse (Diptera: Simuliidae). I. Sibling speciation. Chromosoma 64: 37-65.

BEDo D.G. 1978: Band and nucleolar polymorphisms in polytene chromosomes of Simulium ornatipes (Diptera, Simuliidae). Cytobios 21: 113-133.

BEDo D.G. 1989: A cytological study of Simulium ruficorne (Diptera: Simuliidae) and its relationship to the $S$. ornatipes species complex. - Genome 32: 570-579.

BrockHouse C. 1985: Sibling species and sex chromosomes in Eusimulium vernum (Diptera: Simuliidae). - Can. J. Zool. 63: 2145-2161.

Brockhouse C., Bass J.A.B., Feraday R. \& Straus N. 1989: Supernumerary chromosome evolution in the Simulium vernum group (Diptera: Simuliidae). — Genome 32: 516-521.

Chubareva L.A. \& Petrova N.A. 2008: Tsitologicheskie Karty Politennykh Khromosom i Nekotorye Morfologicheskie Osobennosti Krovososushchikh Moshek Rossii i Sopredel'nykh Stran (Diptera: Simuliidae): Atlas [Cytological Maps of Polytene Chromosomes and Some Morphological Features of Bloodsucking Black Flies of Russia and Adjacent Countries (Diptera: Simuliidae): Atlas.] Tovarishchestvo Nauchnykh Izdanii KMK, St. Petersburg, 134 pp. +218 plates [in Russian.]

Golini V.I. \& Rothrels K. 1984: The polytene chromosomes of North American blackflies in the Eusimulium canonicolum group (Diptera: Simuliidae). — Can. J. Zool. 62: 2097-2109.

Grenier P. \& Dorier A. 1959: Deux Simulies nouvelles (S. bertrandi $\mathrm{n}$. sp. et $S$. carthusiense n. sp.) du groupe latipes, récoltées en France et en Espagne. - Trav. Lab. Hydrobiol. Pisc. Univ. Grenoble 49/50: 205-223.

HUNTER F.F. 1987: Cytotaxonomy of four European species in the Eusimulium vernum group (Diptera: Simuliidae). - Can. J. Zool. 65: 3102-3115.

Hunter F.F. \& Connolly V. 1986: A cytotaxonomic investigation of seven species in the Eusimulium vernum group (Diptera: Simuliidae). - Can. J. Zool. 64: 296-311.

Ilmonen J., Adler P.H., Malmevist B. \& Cywinska A. 2009: The Simulium vernum group (Diptera: Simuliidae) in Europe: multiple character sets for assessing species status. - Zool. J. Linn. Soc. 156: 847-863.

KNOZ J. 1965: To identification of Czechoslovakian black-flies (Diptera, Simuliidae). - Folia Fac. Sci. Nat. Univ. Purkyn. Brun. (Biol.) 6: 1-54.

Rothfels K.H. 1979: Cytotaxonomy of black flies (Simuliidae). - Annu. Rev. Entomol. 24: 507-539.

Rothfels K.H. 1989: Speciation in black flies. Genome 32: 500509 . 
Rothfels K.H. \& Dunbar R.W. 1953: The salivary gland chromosomes of the black fly Simulium vittatum Zett. - Can. J. Zool. 31: 226-241.

Rothfels K.[H.] \& Featherston D. 1981: The population structure of Simulium vittatum (Zett.): the IIIL-1 and IS-7 sibling species. - Can. J. Zool. 59: 1857-1883.

Rothfels K.[H.], Feraday R. \& Kaneps A. 1978: A cytological description of sibling species of Simulium venustum and
S. verecundum with standard maps for the subgenus Simulium Davies [sic] (Diptera). — Can. J. Zool. 56: 1110-1128.

Seitz G. \& Adler P.H. 2009: A new species of the Simulium vernum group (Diptera: Simuliidae) from the Alps of southeastern Germany. - Aquat. Insects 31: 1-10.

Received March 22, 2014; revised and accepted July 11, 2014 Prepublished online August 19, 2014 